\title{
Growth and In Situ Characterization of Oxide Epitaxial Heterostructures with Atomic Plane Precision
}

Q. He, ${ }^{1}$ S. Jesse, ${ }^{2,3}$ A.R. Lupini, ${ }^{1,3}$ M. Fuentes-Cabrera ${ }^{2,4}$, B.G. Sumpter, ${ }^{2,4}$ A. Akbashev, ${ }^{5}$ M. Falmbigl, ${ }^{5}$ J. Spanier, ${ }^{5}$ S. V. Kalinin, ${ }^{2,3}$ and A.Y. Borisevich ${ }^{1,2,3}$

1. Materials Sciences and Technology Division, Oak Ridge National Laboratory, Oak Ridge, TN

2. The Center for Nanophase Materials Science, Oak Ridge National Laboratory, Oak Ridge, TN

3. Institute for Functional Imaging of Materials, Oak Ridge National Laboratory, Oak Ridge, TN

4. Computer Science and Mathematics Division, Oak Ridge National Laboratory, Oak Ridge, TN

5. Department of Materials Science and Engineering, Drexel University, Philadelphia, PA

Manipulation and control the matter at the atomic level is one of the ultimate goals in nanoscience. Ebeam lithography in scanning electron microscope (SEM) geometry ${ }^{[1]}$ have been demonstrated to fabricate three dimensional structures at the nanometer scale. However atomic-level fabrication is only expected with highly energetic e-beams in (scanning) transmission electron microscope ((S)TEM). Recently in-situ fabrication of metallic nanowires of $2 \mathrm{D} \mathrm{MoS}_{2}$ and $\mathrm{MoSe}_{2}$ materials have been demonstrated. However, there are very few reported studies of fabricating 3D material in (S)TEM. For several materials it has been shown that an amorphous area several tens of $\mathrm{nm}$ in size can be converted into a polycrystal ${ }^{[2]}$ or single crystal. ${ }^{[3]}$ Recently, atomic rearrangements in amorphous materials have been reported. ${ }^{[4]}$ These observations suggest that (S)TEM beam can in principle be used to achieve sub-nanometer level bulk nanofabrication.

In our recent work, ${ }^{[5]}$ we demonstrated atomic-level sculpting of $3 \mathrm{~d}$ crystalline oxide nanostructures from metastable amorphous precursor in a scanning transmission electron microscope (STEM). $\mathrm{SrTiO}_{3}$ nanowires were fabricated epitaxially from the crystalline substrate following the beam path. This method can be used for producing crystalline structures as small as 1-2 $\mathrm{nm}$ and the process can be observed in situ with atomic resolution. Tthe nucleation of the crystal happens at the interface between the amorphous film and the crystalline substrate, and the new crystal grows epitaxially. Estimates of possible beam heating suggest that the transformation is knock-on in nature. Atomistic molecular dynamics (MD) simulations verified the feasibility of beam-induced crystallization if the high-energy excitation is applied in the vicinity of the amorphous-crystalline interface. We further demonstrated fabrication of arbitrary shape structures (Fig. 1) via control of the position and scan speed of the electron beam.

In this work, we demonstrate the application of this approach for design and characterization of heterostructures. For precursors we have used amorphous oxide films deposited at low temperature onto crystalline $\mathrm{SrTiO}_{3}$ substrates using atomic layer deposition (ALD). ${ }^{6]}$ Fig.2 demonstrates $\mathrm{BaTiO}_{3} / \mathrm{SrTiO}_{3}$ heterostructures formed using this approach. We will further discuss the dependence of the maximum coherent feature size on the substrate/film mismatch and using this growth approach as a platform for the studies of size-dependent structural transitions. [7]

\section{References:}

[1] C. Vieu et al, Applied Surface Science 2000, 164, 111-117.

[2] I. Jencic et al , Journal of Applied Physics 1995, 78, 974-982; bA. Meldrum, L. A. Boatner, R. C. Ewing, Journal of Materials Research 1997, 12, 1816-1827. 
[3] (a) Y. Zhang et al, Physical Review B 2005, 72, 094112; (b) G. Zhu, G. A. Botton, in The 15th European Microscopy Congress, Manchester Central, United Kingdom, 2012.

[4] K. Zheng et al. Nat Commun 2010, 1, 24.

[5] Jesse, S., et al., Small, 11: 5895-5900 (2015).

[6] S.M. George, Chem. Rev. 110, 111-131 (2010)

[7] The research is sponsored by the Division of Materials Sciences and Engineering, Office of Basic Energy Sciences, U.S. Department of Energy. BGS and MF-C were supported by the Center for Nanophase Materials Sciences which is sponsored at Oak Ridge National Laboratory by the Office of Science, Basic Energy Sciences, U.S. Department of Energy. Calculations made use of resources at the Oak Ridge Leadership Computing Facility at the Oak Ridge National Laboratory, which is supported by the Office of Science of the U.S. Department of Energy under Contract DE-AC05-00OR22725.

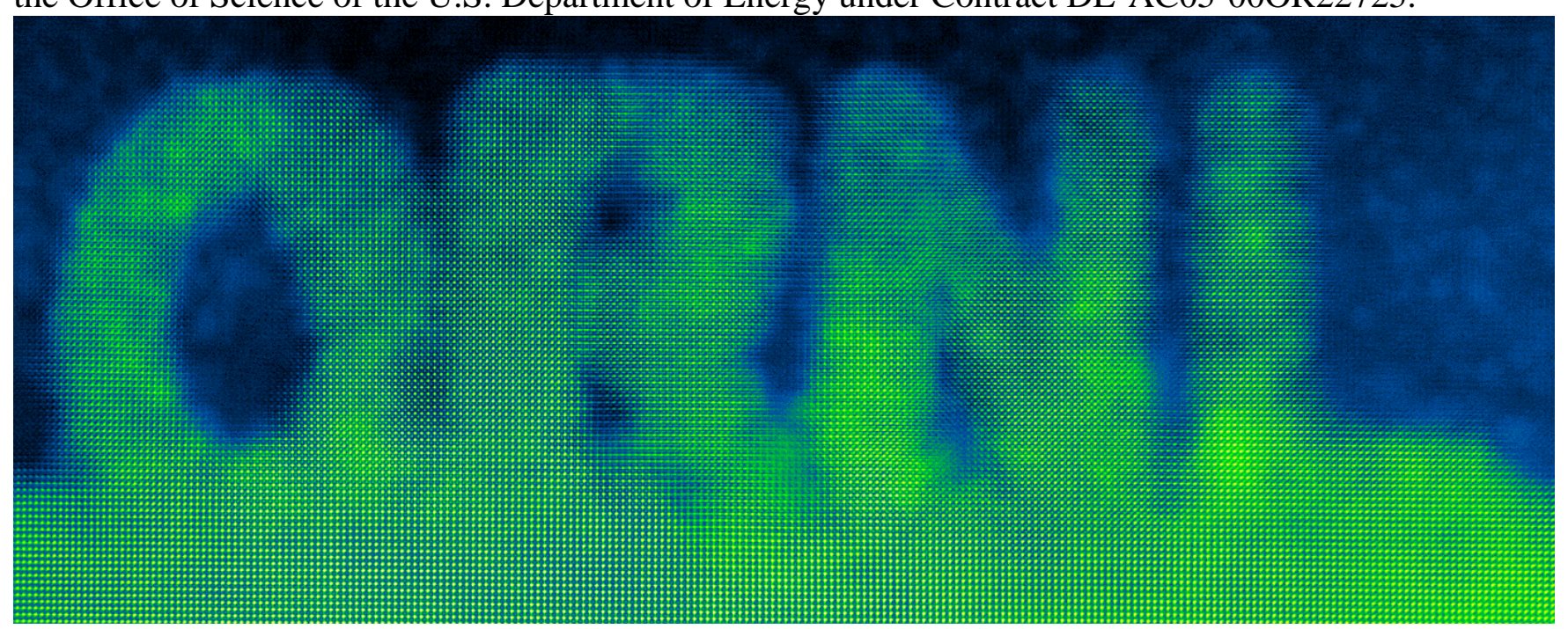

Figure 1. HAADF-STEM image of a fabricated structure showing letters "ORNL" written in crystalline STO using STEM beam. Note that epitaxial alignment with the substrate that is preserved throughout the fabricated struc

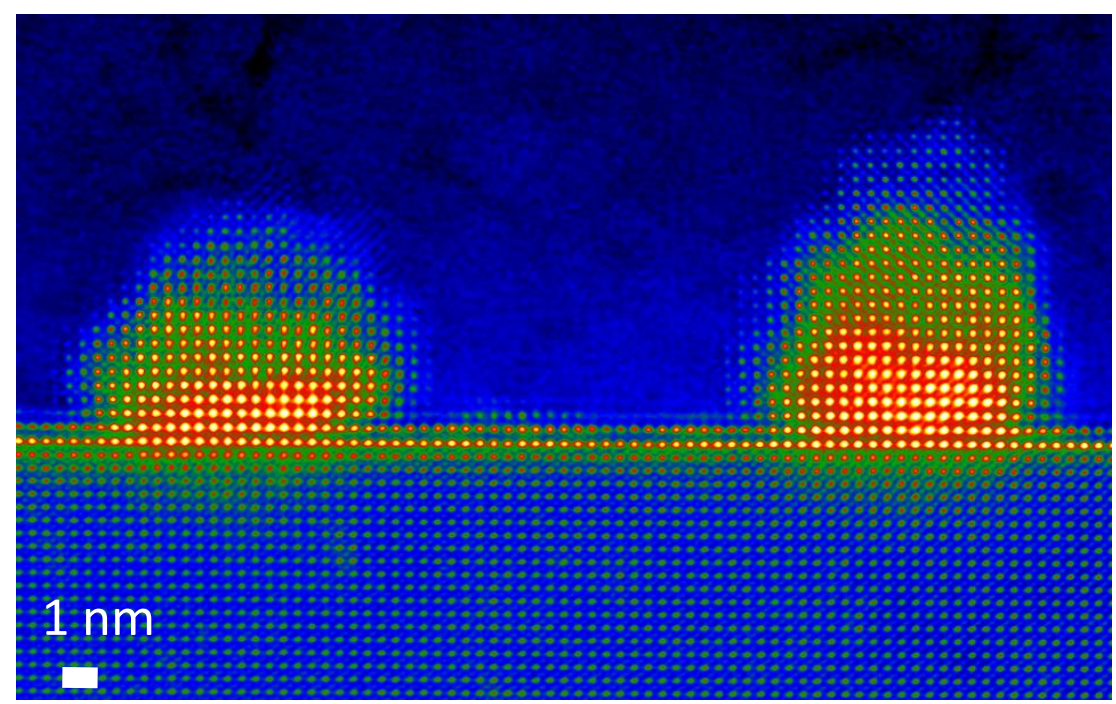

Figure 2. HAADF-STEM image of two $\mathrm{BaTiO}_{3} / \mathrm{SrTiO}_{3}$ heterostructures fabricated from amorphous film using ebeam sculpting approach. Note the abrupt character of the formed interface. 